\title{
A common fixed point theorem for two weakly compatible pairs in $G$-metric spaces using the property E.A
}

\author{
Saurabh Manro ${ }^{1 *}$, Satwinder Singh Bhatia', Sanjay Kumar² and Calogero Vetro ${ }^{3}$
}

"Correspondence:

sauravmanro@hotmail.com

${ }^{1}$ School of Mathematics and

Computer Applications, Thapar

University, Patiala, Punjab, India

Full list of author information is

available at the end of the article

\begin{abstract}
In view of the fact that the fixed point theory provides an efficient tool in many fields of pure and applied sciences, we use the notion of the property E.A to prove a common fixed point theorem for weakly compatible mappings. The presented results are applied to obtain the solution of an integral equation and the bounded solution of a functional equation arising in dynamic programming.

MSC: $47 \mathrm{H} 10 ; 54 \mathrm{H} 25$
\end{abstract}

Keywords: common fixed point; G-metric space; property E.A; weak compatibility

\section{Introduction}

Inspired by the fact that the metric fixed point theory provides an efficient tool in many fields of pure and applied sciences, many authors investigated the possibility to generalize the notion of a metric space. In this direction, Gahler $[1,2]$ introduced the notion of a 2-metric space, while Dhage [3] introduced the concept of a $D$-metric space. Later on, Mustafa and Sims [4] showed that most of the results concerning Dhage's $D$-metric spaces are invalid. Therefore, they introduced a new notion of a generalized metric space, called G-metric space. After then, many authors studied fixed and common fixed points in generalized metric spaces; see [4-15].

Here, we give preliminaries and basic definitions which are helpful in the sequel. First, we introduce the concepts of a G-metric and a G-metric space.

Definition 1.1 [4] Let $X$ be a nonempty set and $G: X \times X \times X \rightarrow[0,+\infty)$ be a function satisfying the following properties:

(G1) $G(x, y, z)=0$ if $x=y=z$;

(G2) $0<G(x, x, y)$ for all $x, y \in X$ with $x \neq y$;

(G3) $G(x, x, y) \leq G(x, y, z)$ for all $x, y, z \in X$ with $z \neq y$;

(G4) $G(x, y, z)=G(x, z, y)=G(y, z, x)=\cdots$ (symmetry in all three variables);

(G5) $G(x, y, z) \leq G(x, a, a)+G(a, y, z)$ for all $x, y, z, a \in X$ (rectangle inequality).

Then the function $G$ is called a generalized metric or, more specifically, a $G$-metric on $X$, and the pair $(X, G)$ is called a $G$-metric space.

Definition 1.2 A $G$-metric space $(X, G)$ is said to be symmetric if $G(x, y, y)=G(y, x, x)$ for all $x, y \in X$.

o 2013 Manro et al: licensee Springer. This is an Open Access article distributed under the terms of the Creative Commons Attribution License (http://creativecommons.org/licenses/by/2.0), which permits unrestricted use, distribution, and reproduction in any medium, provided the original work is properly cited. 
Example 1.3 Let $X=\{2,3\}$ and $G: X \times X \times X \rightarrow[0,+\infty)$ be defined by $G(2,2,2)=$ $G(3,3,3)=0, G(2,2,3)=G(2,3,2)=G(3,2,2)=1, G(2,3,3)=G(3,2,3)=G(3,3,2)=2$. It is easy to show that the function $G$ satisfies all properties of Definition 1.1, but $G(x, x, y) \neq$ $G(x, y, y)$ for all $x, y \in X$ with $x \neq y$. Therefore, $G$ is not symmetric.

Definition 1.4 [4] Let $(X, G)$ be a $G$-metric space, and let $\left\{x_{n}\right\}$ be a sequence of points of $X$. A point $x \in X$ is said to be the limit of the sequence $\left\{x_{n}\right\}$ if $\lim _{n, m \rightarrow+\infty} G\left(x, x_{n}, x_{m}\right)=0$, and we say that the sequence $\left\{x_{n}\right\}$ is $G$-convergent to $x$ or $\left\{x_{n}\right\} G$-converges to $x$.

Thus, $x_{n} \rightarrow x$ in a $G$-metric space $(X, G)$ if for any $\varepsilon>0$, there exists $k \in \mathbb{N}$ such that $G\left(x, x_{n}, x_{m}\right)<\varepsilon$ for all $m, n \geq k$.

Proposition 1.5 [4] Let $(X, G)$ be a G-metric space. Then the following are equivalent:

(1) $\left\{x_{n}\right\}$ is G-convergent to $x$;

(2) $G\left(x_{n}, x_{n}, x\right) \rightarrow 0$ as $n \rightarrow+\infty$;

(3) $G\left(x_{n}, x, x\right) \rightarrow 0$ as $n \rightarrow+\infty$.

Definition 1.6 [4] Let $(X, G)$ be a $G$-metric space. A sequence $\left\{x_{n}\right\}$ is called $G$-Cauchy if for every $\varepsilon>0$, there is $k \in \mathbb{N}$ such that $G\left(x_{n}, x_{m}, x_{l}\right)<\varepsilon$ for all $n, m, l \geq k$; that is, $G\left(x_{n}, x_{m}, x_{l}\right) \rightarrow 0$ as $n, m, l \rightarrow+\infty$.

Proposition 1.7 [4] Let $(X, G)$ be a G-metric space. Then the following are equivalent:

(1) the sequence $\left\{x_{n}\right\}$ is G-Cauchy;

(2) for every $\varepsilon>0$, there is $k \in \mathbb{N}$ such that $G\left(x_{n}, x_{m}, x_{m}\right)<\varepsilon$ for all $n, m \geq k$.

Proposition 1.8 [4] Let $(X, G)$ be a G-metric space. Then the function $G(x, y, z)$ is jointly continuous in all three of its variables.

Definition 1.9 [4] A $G$-metric space $(X, G)$ is called $G$-complete if every $G$-Cauchy sequence in $(X, G)$ is $G$-convergent in $(X, G)$.

Proposition 1.10 [4] Let $(X, G)$ be a G-metric space. Then, for any $x, y, z, a \in X$, it follows that

(i) if $G(x, y, z)=0$, then $x=y=z$;

(ii) $G(x, y, z) \leq G(x, x, y)+G(x, x, z)$;

(iii) $G(x, y, y) \leq 2 G(y, x, x)$;

(iv) $G(x, y, z) \leq G(x, a, z)+G(a, y, z)$;

(v) $G(x, y, z) \leq \frac{2}{3}[G(x, y, a)+G(x, a, z)+G(a, y, z)]$;

(vi) $G(x, y, z) \leq G(x, a, a)+G(y, a, a)+G(z, a, a)$.

An interesting observation is that any $G$-metric space $(X, G)$ induces a metric $d_{G}$ on $X$ given by

$$
d_{G}(x, y)=G(x, y, y)+G(y, x, x), \quad \text { for all } x, y \in X \text {. }
$$

Moreover, $(X, G)$ is $G$-complete if and only if $\left(X, d_{G}\right)$ is complete.

It was observed that in the symmetric case $((X, G)$ is symmetric), many fixed point theorems on $G$-metric spaces are particular cases of the existing fixed point theorems in metric 
spaces. This allows us to readily transport many results from the metric spaces into the G-metric spaces.

On the other hand, by reasoning on the properties of the mappings, the practice of coining weaker forms of commutativity to ensure the existence of a common fixed point for self-mappings on metric spaces is still on. To read more in this direction, we refer to [16] and the references therein. Here, for our further use, we recall only the two fundamental notions of 'weakly compatible mappings' and 'property E.A'; see also [17, 18].

In 1976, Jungck [19] introduced the notion of weakly compatible mappings as follows.

Definition 1.11 Let $S$ and $T$ be two self-mappings of a metric space $(X, d)$. Then the pair $(S, T)$ is said to be weakly compatible if they commute at their coincidence points, that is, if $S u=T u$ for some $u \in X$, then $T S u=S T u$.

In 2002, Amari and El Moutawakil [20] introduced a new concept of the property E.A in metric spaces to generalize the concept of noncompatible mappings. Then, they proved some common fixed point theorems.

Definition 1.12 Let $S$ and $T$ be two self-mappings of a metric space $(X, d)$. Then the pair $(S, T)$ is said to satisfy the property E.A if there exists a sequence $\left\{x_{n}\right\}$ in $X$ such that $\lim _{n \rightarrow+\infty} S x_{n}=\lim _{n \rightarrow+\infty} T x_{n}=t$ for some $t \in X$.

Example 1.13 Let $X=[0,+\infty)$. Define $S, T: X \rightarrow X$ by $S x=\frac{3}{4} x$ and $T x=\frac{x}{4}$ for all $x \in X$. Consider the sequence $\left\{x_{n}\right\}=\left\{\frac{1}{n}\right\}$ in $X$. Clearly, $\lim _{n \rightarrow+\infty} S x_{n}=\lim _{n \rightarrow+\infty} T x_{n}=0 \in X$, and so $S$ and $T$ satisfy the property E.A.

Example 1.14 Let $X=[2,+\infty)$. Define $S, T: X \rightarrow X$ by $S x=2 x+1$ and $T x=x+1$ for all $x \in X$. Suppose that the property E.A holds. Then there exists a sequence $\left\{x_{n}\right\}$ in $X$ such that $\lim _{n \rightarrow+\infty} S x_{n}=\lim _{n \rightarrow+\infty} T x_{n}=t$ for some $t \in X$. It follows that $\lim _{n \rightarrow+\infty} x_{n}=\frac{t-1}{2}$ and $\lim _{n \rightarrow+\infty} x_{n}=t-1$ and so, by Definition 1.12, $t=1$ but $t \notin X$. Therefore, $S$ and $T$ do not satisfy the property E.A.

In conclusion of this preliminary section, we consider the following set:

Let $\Phi$ denote the set of all the functions $\varphi:[0,+\infty) \rightarrow[0,+\infty)$ such that:

(1) $\varphi$ is non-decreasing;

(2) $\lim _{n \rightarrow+\infty} \varphi^{n}(r)=0$ for all $r \in[0,+\infty)$.

If $\varphi \in \Phi$, then it is an easy matter to show that $\varphi(0)=0$ and $\varphi(r)<r$ for all $r \in(0,+\infty)$; see Matkowski [21].

In this paper, by merging the above notions, we prove a common fixed point theorem for two pairs of weakly compatible mappings in a G-metric space. The presented results are applied to obtain the solution of an integral equation and the bounded solution of a functional equation arising in dynamic programming.

\section{Main results}

The following is the main result of this section.

Theorem 2.1 Let $(X, G)$ be a $G$-metric space and $A, B, S, T: X \rightarrow X$ be four self-mappings such that: 
(i) $A(X) \subseteq T(X)$ and $B(X) \subseteq S(X)$;

(ii) one of the pairs $(A, S)$ and $(B, T)$ satisfies the property E.A;

(iii) for all $x, y \in X, G(A x, B y, B y) \leq \varphi(\max \{G(S x, T y, T y), G(S x, B y, B y), G(T y, B y, B y)\})$, where $\varphi \in \Phi$;

(iv) one of $A(X), B(X), S(X)$ and $T(X)$ is a complete subset of $X$.

Then the pairs $(A, S)$ and $(B, T)$ have a coincidence point. Further, if $(A, S)$ and $(B, T)$ are weakly compatible, then $A, B, S$ and $T$ have a unique common fixed point in $X$.

Proof Suppose the pair $(B, T)$ satisfies the property E.A. Then there exists a sequence $\left\{x_{n}\right\}$ in $X$ such that $\lim _{n \rightarrow+\infty} B x_{n}=\lim _{n \rightarrow+\infty} T x_{n}=t$ for some $t \in X$. Since $B(X) \subseteq S(X)$, there exists a sequence $\left\{y_{n}\right\}$ in $X$ such that $B x_{n}=S y_{n}$. Hence $\lim _{n \rightarrow+\infty} S y_{n}=t$. We will show that $\lim _{n \rightarrow+\infty} A y_{n}=t$. From (iii), we have

$$
G\left(A y_{n}, B x_{n}, B x_{n}\right) \leq \varphi\left(\max \left\{G\left(S y_{n}, T x_{n}, T x_{n}\right), G\left(S y_{n}, B x_{n}, B x_{n}\right), G\left(T x_{n}, B x_{n}, B x_{n}\right)\right\}\right) .
$$

Taking the limit as $n \rightarrow+\infty$ (upper limit) and using the fact that $\varphi(r)$ is continuous at $r=0$, we get

$$
\lim _{n \rightarrow+\infty} G\left(A y_{n}, t, t\right) \leq \varphi(\max \{G(t, t, t), G(t, t, t), G(t, t, t)\})=\varphi(0)=0
$$

and so $\lim _{n \rightarrow+\infty} A y_{n}=t$. Thus, we have $\lim _{n \rightarrow+\infty} A y_{n}=\lim _{n \rightarrow+\infty} B x_{n}=\lim _{n \rightarrow+\infty} S y_{n}=$ $\lim _{n \rightarrow+\infty} T x_{n}=t$. Suppose that $S(X)$ is a complete subset of $X$. Then $t=S u$ for some $u \in X$. Now, we will show that $A u=S u=t$. From (iii), we have

$$
G\left(A u, B x_{n}, B x_{n}\right) \leq \varphi\left(\max \left\{G\left(S u, T x_{n}, T x_{n}\right), G\left(S u, B x_{n}, B x_{n}\right), G\left(T x_{n}, B x_{n}, B x_{n}\right)\right\}\right) .
$$

Taking the limit as $n \rightarrow+\infty$, by the property of $\varphi$, we get

$$
G(A u, S u, S u) \leq \varphi(\max \{G(t, t, t), G(t, t, t), G(t, t, t)\})=\varphi(0)=0,
$$

which implies $A u=S u$. Therefore, $u$ is a coincidence point of the pair $(A, S)$. The weak compatibility of $A$ and $S$ implies that $A S u=S A u$ and hence $A A u=A S u=S A u=S S u$. Since $A(X) \subseteq T(X)$, there exists $v \in X$ such that $A u=T v$. We claim that $T v=B v$. Suppose not, from (iii) and using the fact that $\varphi(r)<r$, we have

$$
\begin{aligned}
G(A u, B v, B v) & \leq \varphi(\max \{G(S u, T v, T v), G(S u, B v, B v), G(T v, B v, B v)\}) \\
& =\varphi(\max \{0, G(A u, B v, B v), G(A u, B v, B v)\}) \\
& =\varphi(G(A u, B v, B v)) \\
& <G(A u, B v, B v) .
\end{aligned}
$$

This implies that $A u=B v$ and hence $T v=B v$. It follows that also the pair $(B, T)$ has a coincidence point. Thus, we have $A u=S u=T v=B v$.

Now, if $B$ and $T$ are weakly compatible, then we obtain $B T v=T B v=T T v=B B v$ and show that $A u$ is a common fixed point of $A, B, S$ and $T$. For $x=A u$ and $y=v$, from (iii) and the 
property of $\varphi$, we get

$$
\begin{aligned}
G(A A u, A u, A u) & =G(A A u, B v, B v) \\
& \leq \varphi(\max \{G(S A u, T v, T v), G(S A u, B v, B v), G(T v, B v, B v)\}) \\
& =\varphi(\max \{G(A A u, B v, B v), G(A A u, B v, B v), G(B v, B v, B v)\}) \\
& =\varphi(\max \{G(A A u, B v, B v), G(A A u, B v, B v), 0\}) \\
& =\varphi(G(A A u, B v, B v)) \\
& <G(A A u, B v, B v),
\end{aligned}
$$

which implies $A u=A A u=B v$. Therefore, $A u=A A u=S A u$ is a common fixed point of $A$ and $S$. Similarly, one can prove that $B v$ is a common fixed point of $B$ and $T$. Since $A u=B v$, we deduce that $A u$ is a common fixed point of $A, B, S$ and $T$. Now, we have only to show that the common fixed point is unique. Suppose to the contrary that $w$ and $z$, with $w \neq z$, are two common fixed points of $A, B, S$ and $T$. Then, from (iii) and the property of $\varphi$, we have

$$
\begin{aligned}
G(w, z, z) & =G(A w, B z, B z) \\
& \leq \varphi(\max \{G(S w, T z, T z), G(S w, B z, B z), G(T z, B z, B z)\}) \\
& =\varphi(\max \{G(w, z, z), G(w, z, z), G(z, z, z)\}) \\
& =\varphi(G(w, z, z)) \\
& <G(w, z, z)
\end{aligned}
$$

that is a contradiction and so must be $w=z$. Therefore, $A, B, S$ and $T$ have a unique common fixed point. Clearly, proceeding on the foregoing lines, one can obtain the same conclusion in case (instead of $S(X)$ ) one of $A(X), B(X)$ and $T(X)$ is a complete subset of $X$, and in case (instead of $(B, T))(A, S)$ satisfies the property E.A.

If we assume $S=T$ in above Theorem 2.1, we deduce the following result involving three self-mappings.

Corollary 2.2 Let $(X, G)$ be a G-metric space and $A, B, S: X \rightarrow X$ be three mappings such that:

(i) $A(X) \subseteq S(X)$ and $B(X) \subseteq S(X)$;

(ii) one of the pairs $(A, S)$ and $(B, S)$ satisfies the property $E . A$;

(iii) for all $x, y \in X, G(A x, B y, B y) \leq \varphi(\max \{G(S x, S y, S y), G(S x, B y, B y), G(S y, B y, B y)\})$, where $\varphi \in \Phi$;

(iv) one of $A(X), B(X)$ and $S(X)$ is a complete subset of $X$.

Then the pairs $(A, S)$ and $(B, S)$ have a coincidence point. Further, if $(A, S)$ and $(B, S)$ are weakly compatible, then $A, B$ and $S$ have a unique common fixed point in $X$.

Example 2.3 Let $X=[0,2]$ and $G: X \times X \times X \rightarrow[0,+\infty)$ be defined by $G(x, y, z)=$ $\max \{|x-y|,|y-z|,|z-x|\}$ for all $x, y, z \in X$. Define also $A, B, S: X \rightarrow X$ by $A x=1, B x=2-x$ and $S x=x$ for all $x \in X$ and $\varphi:[0,+\infty) \rightarrow[0,+\infty)$ by $\varphi(t)=t / 2$ for all $t \geq 0$. Clearly, 
the hypotheses (i) and (iv) of Corollary 2.2 hold trivially. Moreover, the pair $(A, S)$ satisfies the property E.A. Here we show only that the hypothesis (iii) holds. In fact, for all $x, y \in X$, we have $G(A x, B y, B y)=G(1,2-y, 2-y)=|1-y|, G(S x, S y, S y)=G(x, y, y)=|x-y|$, $G(S x, B y, B y)=G(x, 2-y, 2-y)=|2-x-y|, G(S y, B y, B y)=G(y, 2-y, 2-y)=2|1-y|$, and consequently,

$$
\begin{aligned}
G(A x, B y, B y) & =|1-y| \\
& =\frac{1}{2} 2|1-y|=\varphi(G(S y, B y, B y)) \\
& \leq \varphi(\max \{G(S x, S y, S y), G(S x, B y, B y), G(S y, B y, B y)\}) .
\end{aligned}
$$

Then, by Corollary 2.2, the pairs $(A, S)$ and $(B, S)$ have a coincidence point, that is, $u=1$. Moreover, since $(A, S)$ and $(B, S)$ are weakly compatible, then $u=1$ is the unique common fixed point of $A, B$ and $S$ in $X$.

\section{Applications to integral and functional equations}

In this section we illustrate two useful applications of our presented results. Firstly, we show how it is possible to obtain the solution of an integral equation by applying Corollary 2.2. Let $\Omega=[0,1]$ and $C(\Omega)$ be the space of all the real continuous functions defined on $\Omega$. Obviously, this space endowed with the $G$-metric given by

$$
G(x, y, z)=\sup _{t \in \Omega}|x(t)-y(t)|+\sup _{t \in \Omega}|y(t)-z(t)|+\sup _{t \in \Omega}|z(t)-x(t)|, \quad \text { for all } x, y, z \in C(\Omega)
$$

is a $G$-complete metric space.

Let $p: \Omega \times \mathbb{R} \rightarrow \mathbb{R}$ and $q: \Omega \times \Omega \times \mathbb{R} \rightarrow \mathbb{R}$ be two continuous functions. Consider an integral equation of the following type:

$$
p(t, x(t))=\int_{\Omega} q(t, s, x(s)) d s .
$$

We will prove the following theorem.

Theorem 3.1 Suppose there exists $H: \Omega \times \mathbb{R} \rightarrow[0,+\infty)$ such that:

(i) $H(s, v(t)) \leq \int_{\Omega} q(t, s, u(s)) d s \leq p(s, v(t))$ for all $s, t \in \Omega$;

(ii) $p(s, v(t))-H(s, v(t)) \leq k|p(s, v(t))-v(t)|$, where $k \in(0,1)$.

Then integral equation (3.1) has a solution in $C(\Omega)$.

Proof Define $(A x)(t)=\int_{\Omega} q(t, s, x(s)) d s$ and $(B x)(t)=p(t, x(t))$. Now

$$
\begin{aligned}
G(A x, B y, B y) & =2 \sup _{t \in \Omega}|(A x)(t)-(B y)(t)| \\
& =\sup _{t \in \Omega}\left|p(t, y(t))-\int_{\Omega} q(t, s, x(t)) d t\right| \\
& \leq 2 \sup _{t \in \Omega}|p(t, y(t))-H(t, y(t))| \\
& \leq 2 k \sup _{t \in \Omega}|p(t, y(t))-y(t)|=k G(y, B y, B y) .
\end{aligned}
$$


Thus, all the hypotheses of Corollary 2.2 are satisfied with $S=I_{C(\Omega)}$, the identity mapping on $\Omega$, and $\varphi(r)=k r$ for all $r \geq 0$ and $k \in(0,1)$. Therefore, there is a unique solution of integral equation (3.1) in $C(\Omega)$.

Now, we study the existence and uniqueness of the bounded solution of a functional equation using again Corollary 2.2. Here we assume that $U$ and $V$ are Banach spaces, $W \subseteq U$ is a state space and $D \subseteq V$ is a decision space.

It is well known that the dynamic programming provides useful tools for mathematical optimization and computer programming as well; see [22-24]. In particular, the problem of dynamic programming related to a multistage process reduces to the problem of solving the functional equation

$$
Q(x)=\sup _{y \in D}\{f(x, y)+K(x, y, Q(\tau(x, y)))\}, \quad x \in W
$$

where $\tau: W \times D \rightarrow W, f: W \times D \rightarrow \mathbb{R}, K: W \times D \times \mathbb{R} \rightarrow \mathbb{R}$.

Let $B(W)$ denote the space of all bounded real-valued functions on $W$. Clearly, this space endowed with the $G$-metric given by

$$
\begin{aligned}
& G(h, k, p)=\max \left\{\sup _{x \in W}|h x-k x|, \sup _{x \in W}|k x-p x|, \sup _{x \in W}|p x-h x|\right\}, \\
& \quad \text { for all } h, k, p \in B(W)
\end{aligned}
$$

is a G-complete metric space.

We will prove the following theorem.

Theorem 3.2 Let $K: W \times D \times \mathbb{R} \rightarrow \mathbb{R}$ and $f: W \times D \rightarrow \mathbb{R}$ be two bounded functions and let $A: B(W) \rightarrow B(W)$ be defined by

$$
A(h x)=\sup _{y \in D}\{f(x, y)+K(x, y, h(\tau(x, y)))\}
$$

for all $h \in B(W)$ and $x \in W$. Assume that the following condition holds:

$$
|K(x, y, h x)-K(x, y, k x)| \leq \varphi(|h x-k x|)
$$

where $x \in W, y \in D$ and $\varphi \in \Phi$. Then functional equation (3.2) has a unique bounded solution.

Proof Note that $(B(W), G)$ is a complete $G$-metric space. Let $\varepsilon$ be an arbitrary positive number, $x \in W$ and $h_{1}, h_{2} \in B(W)$, then there exist $y_{1}, y_{2} \in D$ such that

$$
\begin{aligned}
& A\left(h_{1} x\right)<f\left(x, y_{1}\right)+K\left(x, y_{1}, h_{1}\left(\tau\left(x, y_{1}\right)\right)\right)+\varepsilon, \\
& A\left(h_{2} x\right)<f\left(x, y_{2}\right)+K\left(x, y_{2}, h_{2}\left(\tau\left(x, y_{2}\right)\right)\right)+\varepsilon, \\
& A\left(h_{1} x\right) \geq f\left(x, y_{2}\right)+K\left(x, y_{2}, h_{1}\left(\tau\left(x, y_{2}\right)\right)\right), \\
& A\left(h_{2} x\right) \geq f\left(x, y_{1}\right)+K\left(x, y_{1}, h_{2}\left(\tau\left(x, y_{1}\right)\right)\right) .
\end{aligned}
$$


Then from (3.4) and (3.7), it follows easily that

$$
\begin{aligned}
A\left(h_{1} x\right)-A\left(h_{2} x\right) & <K\left(x, y_{1}, h_{1}\left(\tau\left(x, y_{1}\right)\right)\right)-K\left(x, y_{1}, h_{2}\left(\tau\left(x, y_{1}\right)\right)\right)+\varepsilon \\
& \leq\left|K\left(x, y_{1}, h_{1}\left(\tau\left(x, y_{1}\right)\right)\right)-K\left(x, y_{1}, h_{2}\left(\tau\left(x, y_{1}\right)\right)\right)\right|+\varepsilon \\
& \leq \varphi\left(\left|h_{1} x-h_{2} x\right|\right)+\varepsilon .
\end{aligned}
$$

Hence we get

$$
A\left(h_{1} x\right)-A\left(h_{2}\right) x<\varphi\left(\left|h_{1} x-h_{2} x\right|\right)+\varepsilon
$$

Similarly, from (3.5) and (3.6), we obtain

$$
A\left(h_{2} x\right)-A\left(h_{1} x\right)<\varphi\left(\left|h_{1} x-h_{2} x\right|\right)+\varepsilon .
$$

Therefore, from (3.8) and (3.9), we have

$$
\left|A\left(h_{1} x\right)-A\left(h_{2} x\right)\right|<\varphi\left(\left|h_{1} x-h_{2} x\right|\right)+\varepsilon,
$$

which implies

$$
G\left(A\left(h_{1} x\right), A\left(h_{2} x\right), A\left(h_{2} x\right)\right)<\varphi\left(G\left(h_{1} x, h_{2} x, h_{2} x\right)\right)+\varepsilon .
$$

Since the above inequality is true for any $x \in W$ and $\varepsilon>0$ is taken arbitrary, then we conclude immediately that

$$
G\left(A\left(h_{1} x\right), A\left(h_{2} x\right), A\left(h_{2} x\right)\right) \leq \varphi\left(G\left(h_{1} x, h_{2} x, h_{2} x\right)\right) .
$$

Thus, all the hypotheses of Corollary 2.2 are satisfied with $A=B$ and $S=I_{B(W)}$, the identity mapping on $B(W)$. Therefore, functional equation (3.2) has a unique bounded solution.

Competing interests

The authors declare that they have no competing interests.

\section{Authors' contributions}

All authors contributed equally and significantly to writing this manuscript. All authors read and approved the manuscript.

\section{Author details}

'School of Mathematics and Computer Applications, Thapar University, Patiala, Punjab, India. ${ }^{2}$ Deenbandhu Chhotu Ram University of Science and Technology, Murthal, Sonepat, India. ${ }^{3}$ Dipartimento di Matematica e Informatica, Università degli Studi di Palermo, Via Archirafi 34, Palermo, 90123, Italy.

\section{Acknowledgements}

The authors thank the editor and the referees for their useful comments and suggestions. 


\section{References}

1. Gahler, S: 2-metrische Raume and ihre topologische struktur. Math. Nachr. 26, 115-148 (1963)

2. Gahler, S: Zur geometric 2-metriche Raume. Rev. Roum. Math. Pures Appl. 11, 665-667 (1966)

3. Dhage, BC: Generalized metric space and mapping with fixed point. Bull. Calcutta Math. Soc. 84, $329-336$ (1992)

4. Mustafa, Z, Sims, B: A new approach to generalized metric spaces. J. Nonlinear Convex Anal. 7, 289-297 (2006)

5. Abbas, M, Khan, AR, Nazir, T: Coupled common fixed point results in two generalized metric spaces. Appl. Math. Comput. 217, 6328-6336 (2011)

6. Abbas, M, Rhoades, BE: Common fixed point results for noncommuting mapping without continuity in generalized metric spaces. Appl. Math. Comput. 215, 262-269 (2009)

7. Abbas, M, Nazir, T, Vetro, P: Common fixed point results for three maps in G-metric spaces. Filomat 25, 1-17 (2011)

8. Aydi, H, Shatanawi, W, Vetro, C: On generalized weakly G-contraction mapping in G-metric spaces. Comput. Math Appl. 62, 4222-4229 (2011)

9. Di Bari, C, Vetro, P: Common fixed points in generalized metric spaces. Appl. Math. Comput. 218, 7322-7325 (2012)

10. Manro, S, Bhatia, SS, Kumar, S: Expansion mapping theorems in G-metric spaces. Int. J. Contemp. Math. Sci. 5 , 2529-2535 (2010)

11. Mustafa, Z, Sims, B: Some remarks concerning D-metric spaces. In: Proc. Int. Conf. on Fixed Point Theory and Applications, Valencia, Spain, July 2003, pp. 189-198 (2003)

12. Mustafa, Z, Shatanawi, W, Bataineh, M: Existence of fixed point results in G-metric spaces. Int. J. Math. Math. Sci. 2009, Article ID 283028 (2009)

13. Mustafa, Z, Obiedat, H, Awawdeh, F: Some common fixed point theorem for mapping on complete G-metric spaces. Fixed Point Theory Appl. 2008, Article ID 189870 (2008)

14. Saadati, R, Vaezpour, SM, Vetro, P, Rhoades, BE: Fixed point theorems in generalized partially ordered G-metric spaces. Math. Comput. Model. 52, 797-801 (2010)

15. Shatanawi, W: Fixed point theory for contractive mappings satisfying $\Phi$-maps in G-metric spaces. Fixed Point Theory Appl. 2010, Article ID 181650 (2010). doi:10.1155/2010/181650

16. Di Bari, C, Vetro, C: Common fixed point theorems for weakly compatible maps satisfying a general contractive condition. Int. J. Math. Math. Sci. 2008, Article ID 891375 (2008)

17. Gopal, D, Imdad, M, Vetro, C: Common fixed point theorems for mappings satisfying common property (E.A.) in symmetric spaces. Filomat 25, 59-78 (2011)

18. Gopal, D, Imdad, M, Vetro, C: Impact of common property (E.A.) on fixed point theorems in fuzzy metric spaces. Fixed Point Theory Appl. 2011, Article ID 297360 (2011)

19. Jungck, G: Commuting mappings and fixed points. Am. Math. Mon. 16, 261-263 (1976)

20. Aamri, M, El Moutawakil, D: Some new common fixed point theorems under strict contractive conditions. J. Math. Anal. Appl. 270, 181-188 (2002)

21. Matkowski, J: Fixed point theorems for mappings with a contractive iterate at a point. Proc. Am. Math. Soc. 62 , 344-348 (1977)

22. Baskaran, R, Subrahmanyam, PV: A note on the solution of a class of functional equations. Appl. Anal. 22, 235-241 (1986)

23. Bellman, R: Methods of Nonliner Analysis. Vol. II. Mathematics in Science and Engineering, vol. 61. Academic Press, New York (1973)

24. Bellman, R, Lee, ES: Functional equations in dynamic programming. Aequ. Math. 17, 1-18 (1978)

doi:10.1186/1687-1812-2013-41

Cite this article as: Manro et al.: A common fixed point theorem for two weakly compatible pairs in G-metric spaces using the property E.A. Fixed Point Theory and Applications 2013 2013:41.

\section{Submit your manuscript to a SpringerOpen ${ }^{\circ}$ journal and benefit from:}

- Convenient online submission

- Rigorous peer review

Immediate publication on acceptance

- Open access: articles freely available online

- High visibility within the field

- Retaining the copyright to your article 